\title{
Fluorose dentária em incisivos superiores permanentes em crianças de escola pública do Rio de Janeiro, RJ
} Dental fluorosis in upper permanent incisors of
public schoolchildren in Rio de Janeiro, Brazil

\author{
Branca Heloísa de Oliveira e Priscilla Milbourne \\ Departamento de Odontologia Preventiva e Comunitária da Faculdade de Odontologia da \\ Universidade do Estado do Rio de Janeiro. Rio de Janeiro, RJ, Brasil
}

\section{Descritores}

Levantamentos de saúde bucal." Fluorose dentária, epidemiologia." Dentição permanente. Incisivo. Dentifrícios, prevalência. Brasil, epidemiologia.

\section{Keywords}

Dental health surveys. ${ }^{\#}$ Fluorosis dental, epidemiology. ${ }^{\#}$ Dentition, permanent." Incisor. Dentifrices, prevalence. Brazil, epidemiology.

\section{Resumo}

Objetivo

Determinar a prevalência e a intensidade de fluorose dentária em crianças com idade entre 7 e 12 anos.

Métodos

A população de estudo foi constituída por 266 crianças matriculadas em uma escola pública do Município do Rio de Janeiro, RJ. As crianças tinham idades entre 7 e 12 anos e foram selecionadas pelo método de amostragem aleatória simples. Todos os exames foram feitos entre os meses de agosto e dezembro de 1999 por um único examinador treinado e calibrado (Kappa =0,92). Depois da obtenção do consentimento dos pais, as crianças tiveram seus incisivos superiores permanentes inspecionados sob luz natural. Os dentes foram previamente limpos e secos com rolos de algodão. Os critérios de Russel foram empregados, no diagnóstico diferencial, entre fluorose dentária e opacidades decorrentes de outras causas. O índice de Thylstrup e Fejerskov foi utilizado na determinação da intensidade de fluorose.

Resultados

A prevalência de fluorose foi igual a 7,9\% (IC 95\%, 5,0-11,8). A intensidade variou de 1 a 3, sendo que $77 \%$ dos dentes afetados tiveram registros de grau 1 .

Conclusão

A fluorose dentária não se constitui em problema de saúde pública para a população estudada.

\begin{abstract}
Objective

To assess the prevalence and severity of dental fluorosis in children aged 7 to 12 . Methods

The study population comprised 266 children aged 7 to 12 years, enrolled in a public school in the city of Rio de Janeiro, Brazil, selected using a simple random sample method. All examinations were carried out between August and December 1999 by a single trained and calibrated examiner (Kappa $=0.92)$. After obtaining their parents' consent the children had their upper permanent incisors examined under natural light. Their teeth were previously cleaned and dried with cotton rolls. The differential diagnosis between milder forms of dental fluorosis and nonfluoride enamel opacities
\end{abstract}


was made according to Russel's diagnostic criteria. The severity of fluorosis was assessed by the Thylstrup and Fejerskov Index (TF).

\section{Results}

The prevalence of dental fluorosis was 7.9\% (CI 95\%, 5.0-11.8). The severity ranged from 1 to 3 and $77 \%$ of the affected teeth had a TF score of 1 .

\section{Conclusion}

These results suggest that dental fluorosis does not represent a public health problem in this population.

\section{INTRODUÇÃO}

A descoberta das propriedades anticariogênicas dos fluoretos constitui um dos marcos mais importantes da história da odontologia, uma vez que possibilitou o desenvolvimento de medidas eficazes de prevenção e controle da cárie dentária. Contudo, conhece-se o risco de desenvolvimento de fluorose dentária, quando da ingesta de fluoretos em concentrados acima do recomendado.

A fluorose dentária consiste na hipomineralização do esmalte dentário, produzida pela ingestão crônica de fluoretos, durante o período de desenvolvimento dos dentes. Os sinais clínicos da fluorose variam de linhas brancas finas que seguem as periquimáceas do esmalte até um aspecto totalmente opaco e calcário. Manchas castanhas podem aparecer em virtude da absorção de substâncias presentes na dieta. Nas formas mais graves, pode ocorrer, após a erupção, o desprendimento de porções do esmalte. Isto leva ao aparecimento de depressões na superfície do dente. Pelo fato das alterações no esmalte, decorrentes da fluorose, serem produzidas durante o desenvolvimento das estruturas dentárias, há certa simetria no grau em que os dentes homólogos são afetados. ${ }^{6}$

Em diversas partes do mundo, tem-se constatado um aumento da prevalência de fluorose em crianças, independentemente do fato de a cidade possuir água de abastecimento público fluoretana., ${ }^{9,12}$ Esse fenômeno tem sido associado à ingestão indevida de dentifrícios fluoretados, ao uso de suplementos fluoretados na dieta e à crescente utilização do flúor na alimentação de uma forma geral. ${ }^{8}$ Observa-se, porém, que o aumento nos quadros de fluorose tem sido registrado, predominantemente, nas formas leve e moderada, sem implicar risco para a saúde, constituindo apenas um problema de ordem estética. ${ }^{2}$

Dois estudos realizados no Brasil têm indicado aumento na ocorrência de fluorose dentária semelhante ao que tem sido relatado na literatura internacional. Uma pesquisa, envolvendo as cidades paulistas de Piracicaba, com água fluoretada, e Iracemápolis, sem água fluoretada, mostrou que em ambas ocorreu um aumento de $52 \%$ e $41 \%$ na prevalência de fluorose dentária nos anos de 1991 e 1997, respectivamente. ${ }^{13}$ Outra investigação, conduzida em Porto Alegre, RS, verificou aumento de $24 \%$ na prevalência de fluorose dentária, no período de 1987 a 1997, entre crianças de $8-9$ anos de idade. ${ }^{10}$

Tem-se diagnosticado, em pacientes que procuram a Clínica de Odontopediatria da Faculdade de Odontologia da Universidade do Estado do Rio de Janeiro, inúmeros casos de fluorose dentária. Entretanto, em pesquisa realizada nas bases de dados Lilacs e Medline, não foi encontrado nenhum artigo indexado que fornecesse informações a respeito da prevalência de fluorose dentária em crianças residentes no Município do Rio de Janeiro. Considerando que a determinação da prevalência e da gravidade da fluorose é fundamental para definir a sua importância como um problema de saúde pública, e que é bastante conhecido o fato de que os indivíduos que procuram os serviços de saúde apresentam características que os diferem da população geral, decidiu-se investigar a ocorrência desse problema em uma população de escolares da rede pública municipal de ensino. Portanto, o principal propósito do presente estudo foi determinar a prevalência e a intensidade de fluorose dentária, em crianças entre 7 e 12 anos de idade, que freqüentavam uma escola pública no Rio de Janeiro, RJ. Investigou-se a ocorrência também a relação entre a idade de início da utilização de dentifrícios e a ocorrência de fluorose dentária.

\section{MÉTODOS}

A população de estudo foi constituída por 266 crianças de 7 a 12 anos, nascidas entre 1987 e 1991, selecionadas pelo método de amostragem aleatória simples. A determinação do tamanho da amostra foi feita com uma precisão de 0,05 , em um nível de confiança de $95 \%$, uma prevalência de fluorose esperada de 0,20 e o número total de 448 crianças matriculadas na escola dentro da faixa etária predeterminada. Todas as crianças examinadas encontravam-se freqüentando regularmente a escola pública, no período letivo de 1999, e ingressaram na 
pesquisa após a obtenção do consentimento dos pais ou responsáveis. Essa investigação também foi aprovada pelo comitê de ética em pesquisa do Hospital Universitário Pedro Ernesto.

Os exames clínicos foram realizados, no segundo semestre de 1999, por um único profissional. A calibração do examinador foi efetuada por meio de um estudo de diapositivos de indivíduos com graus variados de fluorose e por exames visuais das arcadas dentárias de crianças portadoras dessa alteração. Foi feito um estudo de confiabilidade, ao longo da pesquisa, com um exame repetido em 33 crianças escolhidas aleatoriamente, estando o examinador mascarado quanto ao resultado do primeiro exame (Coeficiente Kappa $=0,92)$. Os escolares foram acomodados em cadeiras escolares comuns dispostas em salas de aula. A limpeza e a secagem dos dentes foram feitas pelas próprias crianças, esfregando rolos de algodão sobre as superfícies vestibulares dos incisivos superiores. O exame visual dos dentes, limpos e secos, foi realizado sob luz natural. Os registros relativos à ocorrência de fluorose foram feitos em ficha elaborada especialmente para esse fim. Foram atribuídos escores apenas às superfícies vestibulares dos quatro incisivos superiores permanentes presentes (dentes-índice). Foram excluídas da amostra crianças cujos dentes-índice possuíam fraturas extensas, restaurações de resina ou bráquetes que dificultassem o diagnóstico, e também aquelas que não apresentavam todos os quatro incisivos superiores permanentes erupcionados. Os critérios descritos por Russe $1^{15}$ foram considerados para o diagnóstico diferencial entre os casos de fluorose leve ou moderada e para as opacidades de esmalte decorrentes de outras causas. Na determinação da intensidade da fluorose, foi empregado o índice TF (Thylstrup e Fejerskov) ${ }_{1}^{16}$ por permitir a correlação do diagnóstico clínico com mudanças histológicas, proporcionando uma classificação mais precisa dos indivíduos pelos diferentes graus de acometimento de fluorose. ${ }^{14}$ Foi considerada portadora de fluorose dentária toda criança que recebeu escore diferente de zero em, pelo menos, um dente-índice.

A determinação da idade em que foi iniciado o uso de dentifrícios por parte das crianças foi feita por meio de um questionário respondido por seus pais ou responsáveis.

A análise dos resultados foi realizada por programas estatísticos Epi Info 6.04 e Stata 5.0. Distribuições de freqüências foram computadas e gráficos, produzidos. $\mathrm{O}$ teste qui-quadrado de Pearson foi empregado na avaliação estatística das distribuições de freqüências bivariadas. O coeficiente Kappa foi utilizado na avaliação do grau de concordância intra-examinador. O teste de Mann-Whitney foi empregado na comparação entre médias.

\section{RESULTADOS}

Do total de 298 indivíduos selecionados, apenas 10 $(3,35 \%)$ se recusaram a participar, e 22 (7,38\%) tiveram de ser excluídos por não possuírem todos os incisivos superiores permanentes erupcionados ou por terem a visualização da superfície vestibular desses dentes dificultada pela presença de restaurações extensas, fraturas ou bráquetes colados.

Das 266 crianças examinadas, $52,6 \%$ eram do sexo feminino, e 47,4\%, do masculino. A Figura mostra o número de crianças examinadas de acordo com a idade. A freqüência de crianças examinadas com 7 e 8 anos foi menor, porque muitas tiveram de ser excluídas da amostra por não possuírem todos os incisivos superiores presentes na boca. A média de idade do grupo de estudo foi igual a 9,93 (desvio-padrão $=1,47$ ).

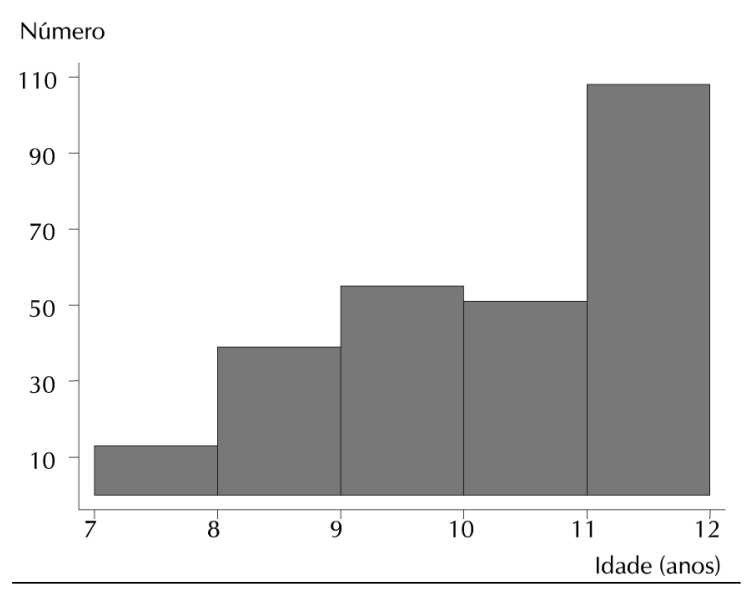

Figura - Histograma que indica o número de crianças examinadas de acordo com a idade. Rio de Janeiro, RJ, 1999.

Verificou-se que $85 \%$ das crianças nasceram e sempre moraram na cidade do Rio de Janeiro. As demais nasceram em outros Estados ou, apesar de serem fluminenses, não viveram por toda a vida nessa cidade.

A prevalência de fluorose dentária entre todas as crianças examinadas foi de $7,9 \%$, com um intervalo de confiança de 95\% (5,0-11,8). Considerando-se apenas as crianças que residiram por toda a vida na cidade do Rio de Janeiro, a prevalência de fluorose obtida foi de $7,1 \%$ (Tabela 1 ). Como não houve diferença significativa entre as duas estimativas $(p=0,49)$, optou-se por conduzir as demais análises considerando-se toda a população de estudo.

A prevalência de fluorose em meninos $(8,73 \%) \mathrm{e}$ meninas $(7,14 \%)$ não apresentou diferença estatis- 
Tabela 1 - Número de crianças examinadas segundo ocorrência de fluorose e residência, por toda a vida, na cidade do Rio de Janeiro, RJ, 1999.

\begin{tabular}{lcccr}
\hline Fluorose & Não & Sim & $\begin{array}{c}\text { Sem } \\
\text { informação }\end{array}$ & Total \\
\hline Sim & 4 & 16 & 1 & 21 \\
Não & 27 & 210 & 8 & 245 \\
\hline Total & 31 & 226 & 9 & 266 \\
\hline
\end{tabular}

ticamente significante $(\mathrm{p}=0,63)$, e as idades médias das crianças portadoras ( 9,6 anos) e livres de fluorose (10 anos) não foram significativamente diferentes ( MannWhitney, $\mathrm{p}=0,24)$.

O grau máximo de fluorose encontrado foi 3 . Enquanto, entre os portadores dessa alteração, a intensidade grau 1 foi a mais freqüente $(77 \%)$. Os dentes homólogos foram afetados pela fluorose de maneira semelhante, havendo alta concordância nos graus de intensidade entre os lados esquerdo e direito, com exceção dos graus 2 e 3 nos incisivos laterais, em que a concordância foi menor (Tabela 2).

Em relação à época de início da utilização de dentifrícios, 79,3\% das crianças já os utilizavam antes dos 3 anos de idade (Tabela 3). A prevalência de fluorose dentária, de acordo com a idade de início da prática da escovação com dentifrício, pode ser vista na Tabela 4.

\section{DISCUSSÃO}

A prevalência de fluorose dentária obtida neste estudo foi igual a 7,9\%. Esse número foi muito menor do que os $20 \%$ esperados, que corresponderiam à
Tabela 3 - Número de crianças examinadas segundo idade de início de utilização de dentifrício. Rio de Janeiro, RJ, 1999.

\begin{tabular}{lrr}
\hline Início do uso de dentifrício & $\mathrm{N}$ & $\%$ \\
\hline $0 \vdash 1$ & 49 & 18,4 \\
$1 \vdash 2$ & 112 & 42,1 \\
$2 \vdash 3$ & 50 & 18,8 \\
$3 \vdash+$ & 35 & 13,2 \\
Não respondeu & 20 & 7,5 \\
\hline Total & 266 & 100 \\
\hline
\end{tabular}

prevalência média encontrada freqüentemente em comunidades, onde o teor de flúor na água é próximo ao ideal. $^{12}$

Alguns fatores relacionados ao desenho da pesquisa podem ter contribuído para a menor prevalência de fluorose observada. Em primeiro lugar, deve-se considerar que o exame visual dos dentes foi feito sob luz natural, e sem que os mesmos tivessem sido submetidos previamente à profilaxia, além de serem secos com ar comprimido. Em função disso, dentes comprometidos por fluorose em graus muito leves podem ter sido considerados saudáveis. Em segundo lugar, tem-se de levar em conta que apenas os incisivos superiores permanentes foram examinados. É provável que, se outros elementos dentários tivessem sido incluídos no estudo, um número maior de casos de fluorose teria sido detectado, pois parece haver aumento progressivo na intensidade dessa alteração dos dentes anteriores aos posteriores. ${ }^{16}$ No entanto, a adoção da metodologia descrita, que também foi empregada por outros autores, ${ }^{5,7}$ permitiu reduzir o tempo gasto com exames, eliminar custos relativos a equipamentos e materiais odontológicos, além de aumentar a adesão das crianças ao estudo, já que os exames puderam ser feitos nas próprias salas de aula.

Tabela 2 - Distribuição de número de crianças examinadas segundo índice TF de fluorose dentária nos incisivos superiores permanentes. Rio de Janeiro, RJ, 1999.

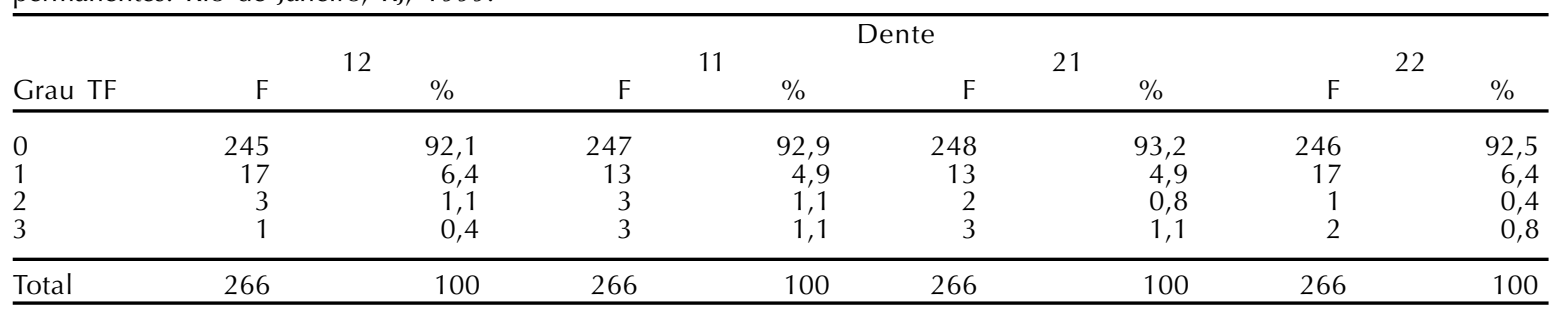

TF: Thylstrup \& Fejerskov ${ }^{16}$

Tabela 4 - Número de crianças examinadas segundo ocorrência de fluorose de acordo com a idade em que foi iniciada a escovação com dentifrício. Rio de Janeiro, RJ, 1999.

\begin{tabular}{|c|c|c|c|c|c|c|}
\hline \multirow{2}{*}{$\begin{array}{l}\text { Início do uso de } \\
\text { dentifrício }\end{array}$} & \multicolumn{2}{|c|}{ Ausência de fluorose } & \multicolumn{2}{|c|}{ Presença de fluorose } & \multicolumn{2}{|c|}{ Total } \\
\hline & $\mathrm{N}$ & $\%$ & $N$ & $\%$ & $\mathrm{~N}$ & $\%$ \\
\hline $0 \vdash 1$ & 46 & $93,9 \%$ & 3 & $6,1 \%$ & 49 & $100 \%$ \\
\hline $1 \vdash 2$ & 100 & $89,3 \%$ & 12 & $10,7 \%$ & 112 & $100 \%$ \\
\hline $2 \vdash 3$ & 50 & $100 \%$ & 0 & 0 & 50 & $100 \%$ \\
\hline $3 \vdash 4$ & 30 & $85,7 \%$ & 5 & $14,3 \%$ & 35 & $100 \%$ \\
\hline Não respondeu & 19 & $95 \%$ & 1 & $5 \%$ & 20 & $100 \%$ \\
\hline Total & 245 & $92,1 \%$ & 21 & $7,9 \%$ & 266 & $100 \%$ \\
\hline
\end{tabular}


Uma pesquisa feita por Clark \& Berkowitz ${ }^{3}$ com crianças canadenses mostrou que, quando toda a dentição era levada em consideração, encontrava-se uma prevalência de fluorose dentária igual a $46 \%$ e que, quando apenas os incisivos superiores eram avaliados, esse número diminuía para $40 \%$. Com base nesses achados, é possível supor que a decisão de examinar apenas incisivos não deve ter tido impacto importante sobre a prevalência de fluorose estimada no presente estudo. Além disso, se for considerado que a implicação mais importante da fluorose dentária nos graus menos intensos, como os observados na presente pesquisa, é de ordem estética, concluise que a proporção de indivíduos de uma comunidade com incisivos superiores afetados por esta condição é que realmente indicará a dimensão da fluorose dentária como problema de saúde pública na população. Portanto, ainda que possa ter ocorrido uma subestimação da prevalência de fluorose dentária na população de estudo, as estimativas obtidas parecem ser suficientes para fornecer uma idéia da importância desse problema nessa comunidade.

Pode-se presumir que o valor encontrado reflete a experiência de fluorose dos escolares de 7 a 12 anos de idade matriculados na escola estudada por ser improvável que tenha ocorrido erro sistemático na seleção dos participantes ou na aferição da ocorrência de fluorose. Já a aferição da presença da fluorose, feita por um examinador capacitado, demonstrou resultados reproduzíveis de forma consistente. Não houve motivos para suspeitar que a população de estudo tenha estado sujeita, dos 15 aos 30 meses de idade, a uma ingestão de fluoretos substancialmente diferente daquela experimentada por crianças da mesma idade que freqüentam outras escolas públicas, localizadas na cidade do Rio de Janeiro. Portanto, sendo a ingestão de fluoretos, naquele período da vida, o fator determinante para o desenvolvimento de fluorose dentária em incisivos superiores, é possível que o alcance dos resultados obtidos por essa investigação não esteja limitado, estritamente, aos alunos da escola estudada. Logo, os resultados sugerem que a fluorose dentária não parece ser um problema de saúde pública para a população infantil da cidade do Rio de Janeiro.

É provável que o pequeno número de casos de fluorose dentária encontrado seja devido, fundamentalmente, à menor exposição das crianças estudadas aos fluoretos, em relação a outros grupos populacionais pesquisados no Brasil e no exterior. ${ }^{12,17}$

Segundo Barros et al, ${ }^{1}$ para se alcançar uma relação risco/benefício eficaz, o teor de fluoreto na água deve ser mantido entre $0,7 \mathrm{ppm}$ e $1,2 \mathrm{ppm}$. No entanto, de acordo com Modesto et al, ${ }^{11}$ o controle dos níveis de flúor da água de abastecimento no Município do Rio de Janeiro não tem sido realizado de forma satisfatória. Ao avaliar amostras de água coletadas em 60 pontos diferentes da cidade, os citados pesquisadores concluíram que todos eles apresentavam concentrações de fluoreto abaixo do ideal, em função da temperatura média anual da região. No bairro de Vila Isabel, onde foi feita a presente pesquisa, verificou-se que o teor de flúor na água era igual a $0,18 \mathrm{ppm}$. O resultado obtido nesta investigação foi semelhante ao encontrado por Elwood et al ${ }^{5}$ na cidade de Maceió, em 1993 (7\%), onde a concentração de flúor na água de beber era inferior a 0,01 ppm. Assim, como foi feito, esses citados autores procederam ao exame das superfícies dentárias de incisivos superiores permanentes sob luz natural, empregaram o índice TF e estudaram a prevalência de fluorose em crianças de baixo nível socioeconômico.

A prevalência de fluorose dentária na população estudada foi similar também à observada por Maltz et $\mathrm{al}^{10} \mathrm{em}$ Porto Alegre, RS, em 1987 (9\%). Após dez anos, sem que tenha ocorrido grande alteração na concentração de flúor na água de abastecimento público nesse município (0,10-1,02 ppm F e 8 meses sem fluoretação), os mencionados autores verificaram que a prevalência de fluorose dentária entre crianças na cidade havia chegado a $33 \%$. Isso seria uma indicação de que as mesmas poderiam estar submetidas a uma maior exposição aos fluoretos a partir de outras fontes, conforme tem-se visto em muitas cidades com água fluoretada, em países desenvolvidos. ${ }^{9}$ A elevada disponibilidade de dentifrícios fluoretados, no Brasil, a partir de setembro de 1984, poderia explicar, em grande parte, o aumento na ocorrência de fluorose observado em Porto Alegre, RS, ao longo desses dez anos. Por sua vez, todas as crianças que compuseram a amostra do presente estudo poderiam ser consideradas potencialmente expostas aos dentifrícios fluoretados, pois nasceram numa época em que mais de $90 \%$ do mercado brasileiro de dentifrícios era formado por produtos com flúor. ${ }^{4}$ De fato, não se encontrou diferença estatisticamente significativa entre as médias de idade das crianças com fluorose e daquelas sem essa alteração. Portanto, poderia se esperar que, caso os teores de flúor na água do Rio de Janeiro, RJ, fossem mantidos dentro da faixa de variação considerada ideal, a população pesquisada apresentaria uma prevalência de fluorose com valor mais próximo ao que foi encontrado em Porto Alegre, RS, em 1997. Esses achados contribuem para reforçar a idéia de que a baixa prevalência de fluorose obtida possa estar associada, entre outras coisas, a teores inadequados de flúor na água de abastecimento público da cidade do Rio de Janeiro. Entretanto, essa hipótese precisa ser confirmada por outros estudos 
que tenham por objetivo específico determinar o grau de exposição das crianças cariocas aos fluoretos provenientes de diferentes fontes, durante o período de formação do esmalte dentário.

Deve-se acrescentar também que os resultados encontrados na presente investigação, e os obtidos por Modesto et al, ${ }^{11}$ fornecem evidências suficientes para indicar que seja premente a implantação de um sistema eficiente de vigilância da fluoretação da água de abastecimento na cidade do Rio de Janeiro.

Considerando a importância atribuída atualmente ao uso precoce de dentifrícios fluoretados como fator de risco ao desenvolvimento de fluorose dentária, procurou-se conhecer a idade de início da exposição das crianças aos dentifrícios. Não se encontrou associação estatisticamente significativa entre a idade de início da escovação (menos de 3 anos/a partir de 3 anos) e a ocorrência de fluorose dentária $(\mathrm{p}=0,15)$. $\mathrm{O}$ resultado obtido difere do que seria esperado com base na literatura e pode ser explicado pela dificuldade de se conseguir informações válidas, quando a coleta de dados é feita de forma retrospectiva sob a forma de questionário e, principalmente, quando a informação de interesse diz respeito a acontecimentos que se deram no passado.

O grau 1 de fluorose do índice TF foi o mais freqüentemente atribuído aos incisivos, nos quais foi constatada a presença de fluorose $(77 \%)$. Portanto, é possível concluir que a maior parte das crianças afetadas por essa condição apresentava alterações mínimas na coloração dos seus incisivos, que dificilmente seriam percebidas por um observador leigo.
A forma simétrica com que a fluorose afeta os elementos dentários pôde ser observada na maioria dos casos. Entretanto, nos incisivos laterais que receberam graus 2 e 3 , a concordância foi menor. Isso pode ser atribuído ao fato dos dentes homólogos não serem necessariamente acometidos por esse distúrbio de desenvolvimento na mesma intensidade. ${ }^{16}$ Não se pode descartar, porém, a possibilidade de ter ocorrido erro de diagnóstico, pelo fato das diferenças na aparência clínica dos dentes acometidos por fluorose graus 2 e 3 serem muito sutis e, portanto, difíceis de ser percebidas nas condições em que os exames foram realizados.

Não se avaliaram a percepção das crianças afetadas a respeito da fluorose nem o impacto dessa condição sobre suas vidas. Portanto, pode-se afirmar apenas que, do ponto de vista estritamente normativo (definição profissional de necessidade de tratamento), a fluorose dentária na comunidade estudada não constitui problema que requeira intervenção do dentista. Serão necessários outros estudos sobre o tema para identificar os possíveis impactos da fluorose dentária sobre a vida diária das crianças acometidas por essa condição em diferentes níveis de intensidade.

\section{AGRADECIMENTOS}

Aos professores Vera Campos, Miriam Souchois e José Augusto Mendes Miguel, do Departamento de Odontologia Preventiva e Comunitária da FO/ UERJ, pelos comentários e sugestões; à técnica em higiene dental Maria Rita dos Santos Rittantonio da FO/UERJ, pela colaboração durante a fase de coleta de dados.

\section{REFERÊNCIAS}

1. Barros ERC, Tovo MF, Scapini MFTC. Análise crítica da fluoretação de águas no município de Porto Alegre. Rev Gaúcha Odontol 1990;38:247-54.

2. Clark DC. Appropriate uses of fluorides for children: guidelines from the Canadian Workshop on the Evaluation of Current Recommendations Concerning Fluorides. Can Med Assoc / 1993;149:1787-93.

3. Clark DC, Berkowitz J. The influence of various fluoride exposures on the prevalence of esthetic problems resulting from dental fluorosis. J Public Health Dent 1997;57:144-9.

4. Cury JA. Cárie e creme dental. J ABOPREV 1998;10:2.

5. Ellwood RP, Cortes DF, O'Mullane DM. A photographic study of developmental defects of enamel in Brazilian school children. Int Dent J 1996;46:69-75.

6. Fejerskov O, Baelum V, Manji F, Moller IJ. Fluorose dentária: um manual para profissionais de saúde. São Paulo: Editora Santos; 1994.

7. Gaspar MR, Armbruster LM, Pereira AC, Moreira BW. Opacidades de origem não-fluorótica e fluorose dentária em áreas com baixa $(0,2$ ppm) e ótima $(0,7$ ppm) concentração de flúor na água de abastecimento. Rev Bras Odontol 1995;52:13-8.

8. Horowitz HS. Fluoride and enamel defects. Adv Dent Res 1989;3:143-6.

9. Leverett D. Prevalence of dental fluorosis in fluoridated and nonfluoridated communities: a preliminary investigation. I Public Health Dent 1986;46:184-7. 
10. Maltz M, Silva BB, Schaeffer A. Prevalência de fluorose em uma cidade brasileira com água fluoretada, em 1987 e 1997. In: 15a Reunião Anual da Sociedade Brasileira de Pesquisa Odontológica. Águas de São Pedro (SP); 1998. p. 70.

11. Modesto A, Tanaka FHR, Freitas AD, Cury JA. Avaliação da concentração de fluoreto na água de abastecimento público do Município do Rio de Janeiro. Rev Bras Odontol 1999;56:217-21.

12. Pendrys DG, Stamm JW. Relationship of total fluoride intake to beneficial effects and enamel fluorosis. $J$ Dent Res 1990;69:529-38.

13. Pereira AC, Cunha MC, Meneghim MC. Prevalência de cárie dentária e fluorose em escolares de áreas fluoretadas e não fluoretadas. In: $15^{a}$ Reunião Anual da Sociedade Brasileira de Pesquisa Odontológica. Águas de São Pedro (SP); 1998. p. 121.
14. Richards A, Fejerskov O, Baelum V. Enamel fluoride in relation to severity of human dental fluorosis. $A d v$ Dent Res 1989;3:147-53.

15. Russell AL. The differential diagnosis of fluoride and nonfluoride enamel opacities. J Public Health Dent 1961;21:143-6.

16. Thylstrup A, Fejerskov O. Clinical appearance of dental fluorosis in permanent teeth in relation to histologic changes. Community Dent Oral Epidemiol 1978;6:315-28.

17. Tomita NE, Panighel CPM, Narvai PC, Lopes ES. Implicações da vigilância à saúde sobre a ocorrência de fluorose dental. Rev ABO Nac 1995;3:318-23. 